\title{
Information Quality Aware Data Collection for Adaptive Monitoring of Distribution Grids
}

\author{
Mohammed S. Kemal, Rasmus L. Olsen, and Hans-Peter Schwefel \\ Aalborg University, Denmark \\ seifu, rlo, hps@es.aau.dk
}

\begin{abstract}
Information from existing smart metering infrastructure, which are currently mainly used for billing purposes, can also be utilized to monitor and control state of the grid. To add functionalities such as fault detection and real-time state estimation, data from smart meters should be accessed with increased frequency during run time. The data collection system should adapt to changing dynamics of the communication network and electrical grid. This paper first introduces adaptation functionalities for the data collection mechanism. To study and analyze the influence of configuration parameters that can be utilized for adaptation, a two-layer smart meter data access infrastructure is presented. An information quality metric, Mismatch Probability $(\mathrm{mmPr})$ is introduced for the quantitative analysis of the two-layer data access system implemented in MATLAB based discrete event simulation study.
\end{abstract}

Key words: Adaptive Data Collection, Network QOS, Smart Metering Infrastructures, Distribution Grid Monitoring, Information Quality

\section{Introduction}

In recent years, various initiatives have been proposed to standardize the communication to smart meter units. Examples of standardization and initiatives include, IEC 62056-58, IEC 62056-62 data exchange for meter reading, tariff and load control [5] [6], Device Language Message Specification DLMS/COSEM protocol [7], and communication system for meters and remote reading of meters [8]. The standardizations have opened the door for a better system integration of smart metering infrastructures for applications such as Demand Response and Home Energy Management Systems [9].

Regarding monitoring and automation of electric distribution systems, reference [10] presents a mechanism that utilizes smart metering infrastructure using GSM technology. The developed model is tested using two-way communication for remote control of supply and outage management along with automatic meter switching-off on the occurrence of a fault. References [11], [12] propose a detailed examination of active and non-active power components by making use of modern smart meters for studying power quality, load monitoring and active power factor correction.

Adaptivity of network QoS and information access configurations is studied in great detail in the context of the SmartC2Net project [13], with the primary goal 
of enabling Smart Grid operations over imperfect, heterogeneous general purpose networks. References [14] [15] studied an Information-Quality based low voltage (LV) grid monitoring framework and its application to power quality control. Reference [16] discusses utilizing network QoS for adaptive Smart Grid control with a focus on the influence of imperfect network conditions on Smart Grid controllers, and how this can be counteracted by utilizing Quality of Service (QoS) information from the communication network.

The approach proposed in this paper for monitoring of the electrical grid by adaptive data collection methods is focusing on using smart metering infrastructures with an adaptation of access rates on metering data. Currently, most of the smart meters deployed in Denmark are used for billing purposes with meter readings at slow update rates [17]. The main contribution of this work is to investigate scenarios of access to smart meter data with short time granularity; specifically, the paper evaluates the impact of configurations of access procedures and the influence on the quality of smart meter data for varying communication delays and different scenarios of smart meter data variability. The overall concept of adaptivity is outlined in reference [4], here we go beyond this concept by adding the quantitative analysis of a two layer architecture and analyze dependencies between configuration parameters that can be adapted to the data collection system.

\section{Adaptation Functionalities}

For applications such as grid fault detection and real-time state estimation, the smart meter data is used in real-time with different time-horizons [20]. The update interval varies accordingly ranging from few seconds for grid-state estimation to few minutes for fault-localization. The actual measurement and data transmission frequency of the individual smart meters may, however, depend on various factors, and this paper will analyze benefits of adapting this measurement update frequency during run-time. The following basic adaptation scenarios can be distinguished:

Adaptivity to Changing Grid Dynamics: For an environment with high penetration of unpredictable, shifting solar PV units and storage devices in households where the state of assets changes fast, the grid is susceptible to short time power variations of distributed generations. To give an example, during partially cloudy conditions, PV generation can fluctuate as fast as within a few seconds, influencing part of the LV grid behaviour with similar fast dynamics. If the data collection mechanism can adapt to the changing dynamics of the condition or process, reliable operation can be ensured within the communication constraints. Adapting update rates at smart meters accordingly would give us an improved observability of the distribution grid. However, this occurs at the cost of increased network load, which is addressed in the next paragraph. 
Adaptivity to Changing Communication Network Dynamics: A communication network is one of the core parts of the smart metering data collection scheme. It is also inherent that for both wired (e.g., copper cable, fiber optic cable, and power line carrier) and wireless communications (e.g., cellular, satellite, microwave, and WiMAX), the communication network is variable and changing continually. For example, if the communication medium is shared with other users ( e.g., GSM, 3G, shared PLC), the impact of cross traffic has an effect and changes performance of the whole system creating increased delays, packet losses and low throughput. The data collection scheme should be adaptive to this variability to facilitate grid monitoring functionalities [2]. A two layer architecture is modelled to study and analyse dependencies between the configuration parameters that can be adapted to both changing dynamics of the grid and communication network which will be described in the next section.

\section{Analysis Of Two Level Smart Meter Data Access Infrastructure}

Smart meter data is typically accessed via concentrators that store, aggregate, and forward information from multiple meters. That way, a two-level access structure is resulting which is illustrated in Figure 1 and further described and analysed as follows.

\subsection{System Description}

This section gives an abstracted description of the two level smart meter data access infrastructure (message sequence diagram shown in Figure 1). The two level data access infrastructure contains the following parts:

Change Event Process $E_{i j}$ : The smart meter has capability to access a local measurand (typically power or voltage), which changes value over time. We consider a discretized value space, where only changes beyond a certain discretization threshold are considered. In this discretized setting, we call the time instances $E_{i j}, j=1,2, \ldots$ when the measurand changes value the 'change event process' at Smart Meter i, $i=1, \ldots, N$. We use the same parameter $\lambda$ as rate of this change event process for all $\mathrm{N}$ smart meters.

Smart Meter Update Rate $\tau_{s m}$ : Each smart meter takes a measurement and sends it to the data concentrator with rate $\tau_{s m}$, which we call smart meter update rate.

Concentrator Update Rate $\tau_{a}$ : Similarly the data concentrator sends its current view on the smart meter measurands to the control layer with an update rate of $\tau_{a}$. 


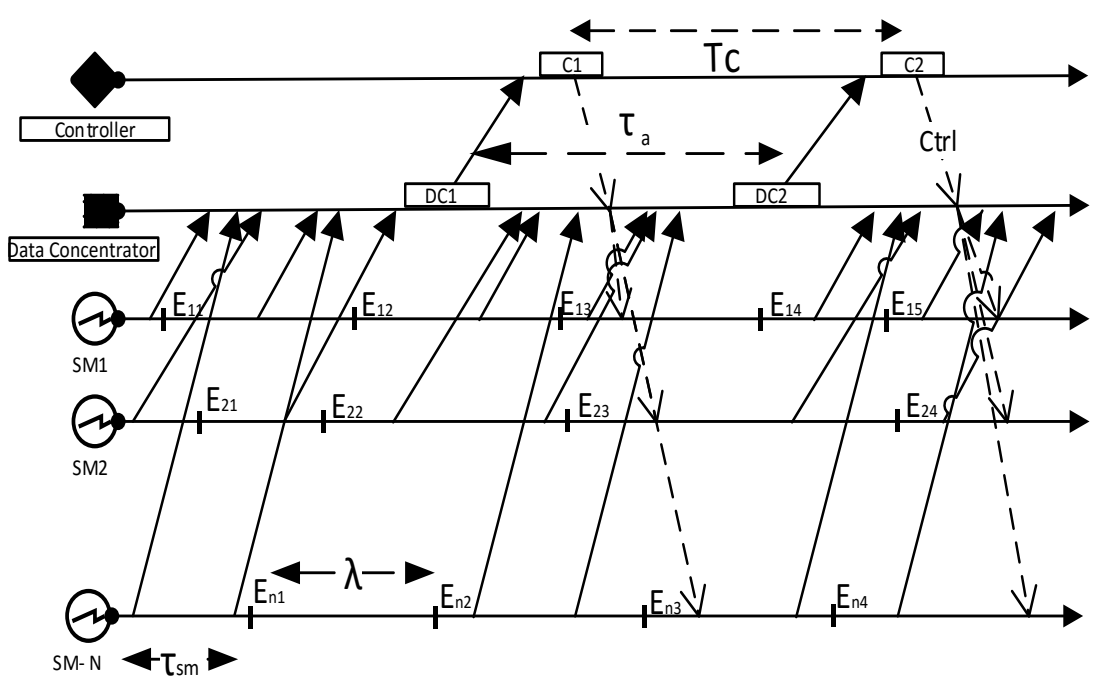

Fig. 1: Two Level Smart Meter Data Access Infrastructure

Control Action Time $C_{k}$ : represent the moments where the controller takes action based on the available information of the smart meter values. We assume here that the time between control actions is deterministic with value $T_{C}$.

Communication Delays $\nu_{s m}$ and $\nu_{a}$ : Communication between each layer is represented by stochastically varying delays with mean values $\nu_{s m}$ and $\nu_{a}$. The communication network is assumed to be reliable by utilizing retransmissions to compensate for losses. The downstream communication from the controller depicted by the dashed arrow is not taken into consideration for the analysis implemented in this paper.

\subsection{Simulation Model and Metrics}

In order to analyze the two-level smart meter data access strategy, we use a stochastic discrete event simulation realized in Matlab. For the simulation studies, the information element at each smart meter is maintained by each smart metering node and dynamically changes its value at certain points with a poisson process. We also make the assumption that the information change is always resulting in a new value not observed previously. The time between information updates is exponentially distributed with rate $\tau_{s m}$ and $\tau_{a}$. A Poisson process is chosen because the focus and interest on this paper is for cases where smart 
meters send updates with some variability, modelled by a stochastic process. The parameter space is simplified by using a poisson process which also gives us flexibility to perform further mathematical analysis, which for now is beyond the scope of this paper. The default assumption for the communication infrastructure is a static network model where the mean values $\nu_{s m}$ and $\nu_{a}$ are assumed to be constant. Later in Section 3.4, this assumption is however modified in order to capture also the impact of the smart metering traffic on the communication network performance. Smart meter and data concentration layers are considered to be symmetric by utilizing the same communication network where $\nu_{s m}=\nu_{a}$.

To study a PLC communication scenario where congested and non congested PLC (with cross traffic) links are used for the two layer data access scenario, mean communication delays of $5 \mathrm{~s}$ and $250 \mathrm{~ms}$ are set respectively (static network assumption). The choices are motivated based on experimental studies also presented by [22]. For analysis of fast changing information elements at the smart meters $\lambda=10 \mathrm{~s}$ is derived for a normal household based on statistical studies for residential load modelling done by [21]. The control interval Tc for all the tests is $2.5 \mathrm{~s}$ motivated by a microgrid control scenario in [22]. All the simulation results presented in this paper are for simulation time of 1000s for each update rate combinations.

The motivation for the smart meter data access is a control scenario as for instance analyzed in [22] for a power balancing controller. However, this paper will stay independent of the specific controller realization and therefore uses information quality metric, which has first been introduced in [19]: The mismatch probability $(\mathrm{mmPr})$ is the probability that the information at the controller, during the time of control execution $C_{i}$ does not match within the discretization thresholds with the true measurand value at the smart meter at that instance (see Equation 1). This is under the hypothesis that a correct and timely information leads to expected control performance, while mismatching information degrades the control performance. The advantage of $\mathrm{mmPr}$ as metric is that it combines in a single scalar the impact of update rate choices and network delays and puts those in relation to the dynamics of the grid scenario (measurand at the smart meter). The metric has been successfully applied to optimize Smart Grid control in [14] and [15]. Equation 1 shows mmPr calculation for a smart meter, where info $o_{\text {controller }}$ denotes the controller's view on the Smart Meter information and info $_{S M}$ denotes the actual value of the measurand at the smart meter; both are considered at actuation moments $C_{k}$.

$$
m m P r=\operatorname{Pr}\left(\text { info }_{\text {controller }}\left(C_{k}\right)\right) \neq \operatorname{info}_{S M}\left(C_{k}\right),
$$

\subsection{Quantitative Analysis of Smart Meter Update Rates Over Static Delay Model}

In this paper, we are mainly interested in the impact of the update rate of the smart meters and of the concentrator on the resulting information quality at the control instances. We therefore vary these update rates in the ranges $0 / s$ to $2 / s$ and $1 /$ lambda of 10s. Figure 2 shows $3 \mathrm{D}$ plot of the resulting mmPr values for 
varying $\tau_{s m}$ and $\tau_{a}$ by using non congested PLC communication. As clearly seen in the relationship, when any of the update rates go to a zero value the $\mathrm{mmPr}$ values approaches to 1 which is clear as less update maps to less knowledge of the current state of the meter (higher mismatch).

A different 2D visualization of the same simulation results in Figure 3 shows the $\tau$-combinations that lead to different levels of mmPr, e.g. any ( $\tau_{s m}$ and $\tau_{a}$ ) combination above and to the right of the blue line with $\mathrm{mmPr}=0.2$ would perform better than that mmPr threshold. It can also be seen in the result that updating $\tau_{a}$ may not necessarily change the $\mathrm{mmPr}$ value on the left top section of the plot, if $\tau_{s m}$ stays constant. A similar observation holds for large $\tau_{s} m$ in the lower right part of the graph.

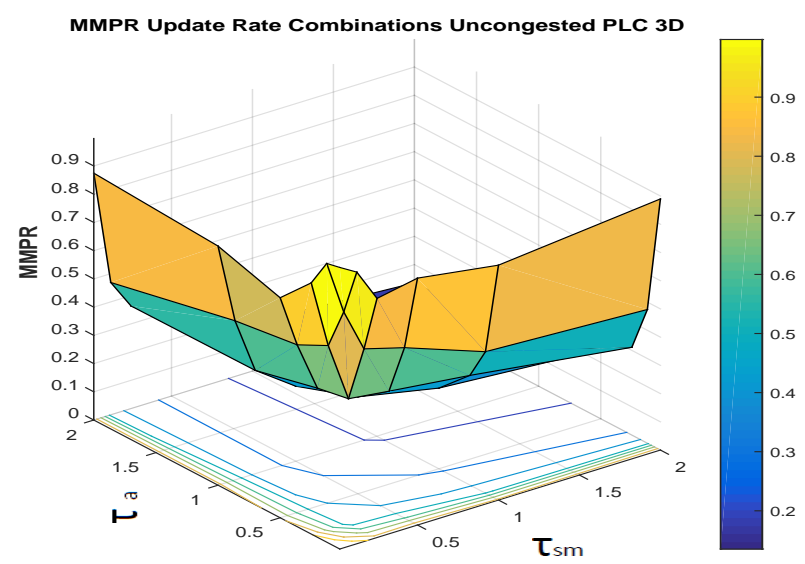

Fig. 2: Update Rate Combinations to fulfil mmPr requirement bounds for non congested PLC scenario 3D

Figure 3 and 4 show the contrast between the congested and non-congested PLC scenarios. Fig 4 shows that the delays are so high that small mismatch probabilities below $50 \%$ are not even reachable even with update periods as small as 0.5 seconds (right and top end of graph). For even higher update rates, the $\mathrm{mmPr}$ would actually decrease further (due to the assumption of i.i.d exponential delays and the resulting order statistic properties of the smallest sample). However, such scenarios are not realistic as eventually the limited communication network bandwidth would lead to increased delays, see Section 3.4. The artifacts shown in Figure 4, for example at the right with $\mathrm{mmPr}$ of 0.7 is due to statistical fluctuation and stochastic nature of the simulations. 


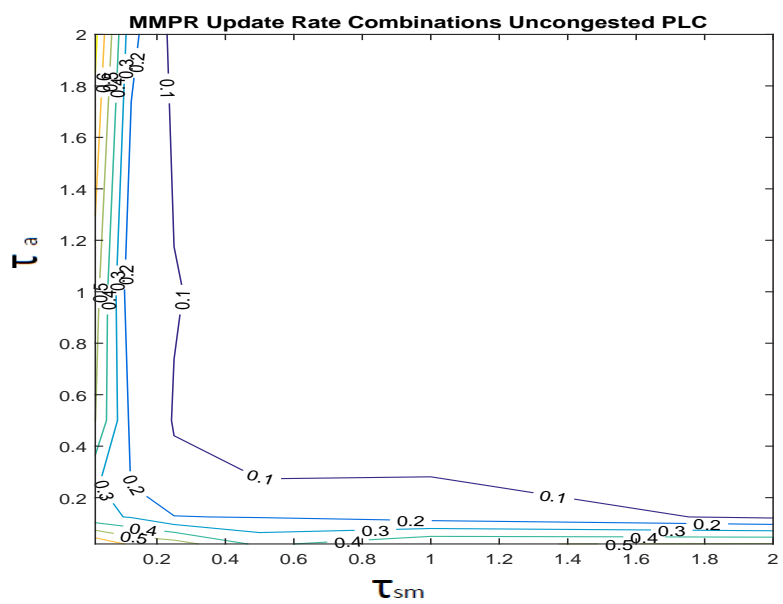

Fig. 3: Update Rate Combinations to fulfil mmPr requirement bounds for non congested PLC scenario

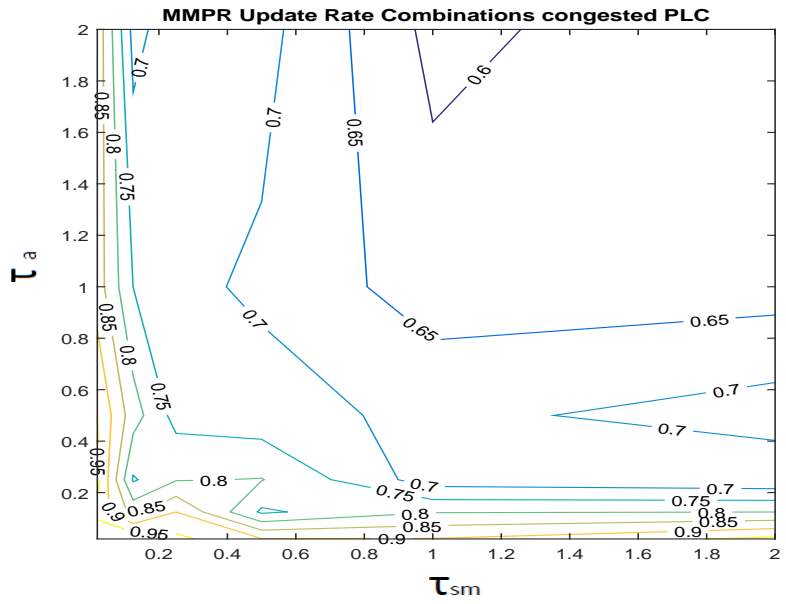

Fig. 4: Update Rate Combinations to fulfil mmPr requirement bounds for congested PLC scenario 


\subsection{Quantitative Analysis of Smart Meter Update Rates Over Update Rate Dependent Delay Model}

To investigate a load dependent delay communication scenario, a linear increase of mean network delays for increasing smart meter update rate is considered next, i.e. $\nu_{s m}=a^{*} \tau_{s m}+b$, where $a$ and $b$ are chosen to maintain a delay range of $0.2 \mathrm{~s}$ to $2.5 \mathrm{~s}$ covering the range for a PLC scenario and $\tau_{s m}$ range of 0.02 to 2 . The choice of linear dependency provides a simplified assumption for analysing the impact of network traffic (increased $\tau_{s m}$ ) on $\nu_{s m}$. For the model of communication network at the data concentrator layer, $\nu_{a}=250 \mathrm{~ms}$ is considered, which is inspired by a $3 \mathrm{G}$ communication scenario [23].

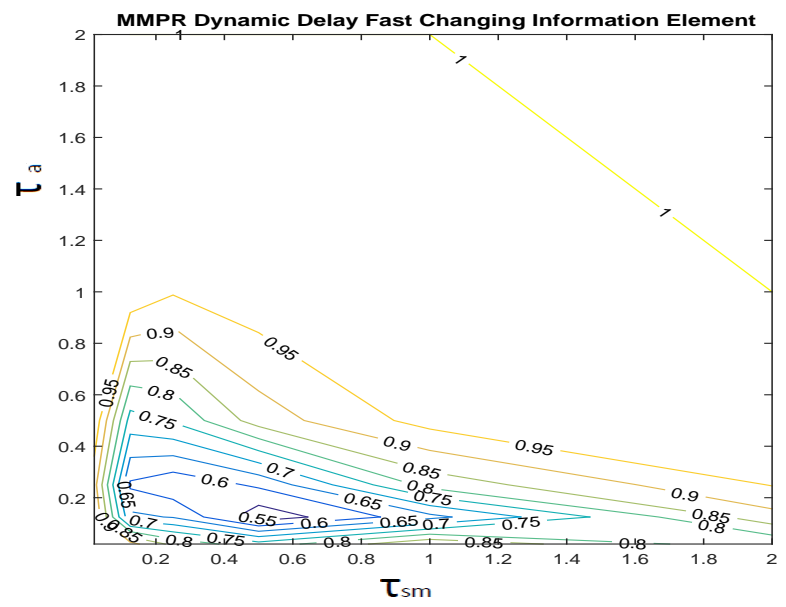

Fig. 5: Update Rate Combinations to fulfil $\mathrm{mmPr}$ requirement bounds for fast changing information element with mean time between information change of 10 s and a linearly growing network delay between smart meter and concentrator for increasing $\tau_{s m}$.

As can be seen in Figure 5, due to the impact of of the linearly dependent network model, update rate combinations of $\tau_{s m}$ of 2 and $\tau_{a}$ of 2 lead to an $\mathrm{mmPr}$ of 1 unlike the Figure 3 and 4 . The circular shape of the $\mathrm{mmPr}$ curves in Figure 5 shows that there is a minimum for the mmPr; the value of the minimum in these simulation results is between 0.5 and 0.55 and it is achieved for $\tau_{s m} \approx 0.46$ and $\tau_{a} \approx 0.17$. To study the impact of change of information element, we have also tested a scenario with $\lambda$ of $1 / 60 s$. The lower $\lambda$ does not seem to impact the update rate combinations to have an absolute minimum of the mmPr in Figure 5 , although the $\mathrm{mmPr}$ values are lower due to the slow changing information element. 


\section{Summary and Outlook}

This article provides a general overview of adaptive data collection functionalities for monitoring of distribution grid through the existing smart metering infrastructure. The results presented in the paper show that update rate configurations at smart meter and data concentration layers can be used to adapt the data collection system. A performance metric mmPr is introduced and used for quantitative analysis of two layer data access infrastructure.

The simulation analysis performed in this paper is based on stochastic assumptions, the future plan is to collect measurement traces from smart meters via measurement campaign and use the measured values to make further quantitative analysis. In addition, procedures for online adaptation of update rates will be developed and tested in Real-Time hardware in the loop (HIL) integration and in field tests.

\section{Acknowledgment}

This work is financially supported by the Danish project RemoteGRID which is a ForsKEL program under Energinet.dk with grant agreement no. 2016-1-12399.

\section{References}

1. J. Zheng and D. W. Gao and L. Lin, Smart Meters in Smart Grid: An Overview, Green Technologies Conference, 4-5, Denver, USA, April 2013.

2. REMOTEGRID - REliable MOniToring and Estimation of distribution GRID for smart societies, [Online], Available: http://www.remotegrid.dk/ 2015.

3. K. D. Craemer and G. Deconinck, Analysis of State-of-the-art Smart Metering Communication Standards, Proceedings of the 5th Young Researchers Symposium, IEEE, 2010.

4. M. Kemal, R. Olsen, Adaptive Data Collection Mechanisms for Smart Monitoring of Distribution Grids, 12th European Dependable Computing Conference (EDCC 2016), Gothenburg, Sweden, (Proceedings of Student Forum), September 5-9, 2016.

5. IEC 62056-58, Data exchange for meter reading, tariff and load control, Part 58: Smart Message Language, 2006.

6. IEC 62056-62, Data exchange for meter reading, tariff and load control. Part 62: Interface Classes, 2006.

7. IEC 62056-53, Data exchange for meter reading, tariff and load control. Part 53: COSEM application layer, 2006.

8. EN 13757, Communication system for meters and remote reading of meters, 2008.

9. S.Mohagheghi and J. Stoupis and Z. Wang and Z. Li and H. Kazemzadeh, Demand Response Architecture: Integration into the Distribution Management System, Smart Grid Communications (SmartGridComm), 2010.

10. N.N. Naik and M.N Dhend, Development of Smart Meter for Distribution System Monitoring, Discovery The International Journal, 2015. 
11. J. Anderson, A. Sadhanala and R. Cox, Power Quality, Smart Meters and Additional Information from Different Power Terms, IEEE Latin America Transactions, 158-165, 2015.

12. W. Angelino de Souza and F. Pinhabel Marafao and E. Verri Liberado and I. Severino Diniz and P. J. Amaral Serni, Using smart meters for load monitoring and active power-factor correction, IECON 2012-38th Annual Conference on IEEE Industrial Electronics Society, 2012.

13. Smart Control of Energy Distribution Grids over Heterogeneous Communication Networks, [Online], Available:"http://www.smartc2net.eu/".

14. M. Findrik, Th. Kristensen, Th. Hinterhofer, R. L. Olsen, H. Schwefel, InformationQuality based LV-Grid-Monitoring Framework and its Application to Power-Quality Control, Ad Hoc Now, June 2015.

15. T. Kristensen, R. Olsen, J. Rasmussen, Analysis of Information Quality in event triggered Smart Grid Control, Vehicular Technology Conference 2015-Spring, May 2015.

16. J. Theilgaard Madsen, T. Le F. Kristensen, R. Olsen, H. Peter Schwefel, L. Cristiana Totu, Utilizing Network QoS for Dependability of Adaptive Smart Grid Control, IEEE Energycon 2014, May 2014.

17. ESSnet Big Data, Work Package 3 : Smart Meters , [Online], Available:"https://webgate.ec.europa.eu".

18. M. Simonov and G. Zanetto, Event-based hybrid metering feeding AMI and $S C A D A$, Event-based Control, Communication, and Signal Processing (EBCCSP), 2015.

19. B. Martin, R. Olsen, H. Peter Schwefel, Probabilistic models for access strategies to dynamic information elements, Performance Evaluation, 67(1), 4360, 2006.

20. Y. Hu, A. Kuh, A. Kavcic, Real-time State Estimation on Micro-grids IEEE Computational Intelligence Magazine, 2011.

21. M.Dong, P.C.Meira, W.Xu, W.Freitas, and A. Brunstrom,An Event Window Based Load Monitoring Technique for Smart Meters, IEEE TRANSACTIONS ON SMART GRID : Vol.3, No.2 2012.

22. R.Pedersen, M.Findrik, Hasenleithner, Eduard, C.Sloth, and H.P.Schwefel, Network Condition Based Adaptive Control and its Application to Power Balancing in Electrical Grids, Sustainable Energy, Grids and Networks, Published, Jun 2017.

23. M.Findrik, R.Pedersen, C.Sloth, and H.P.Schwefel, Test-bed assessment of communication technologies for a power-balancing controller, ENERGYCON 2016, Published, 2016. 